\title{
Surface resonant modes in colloidal photonic crystals
}

\author{
A. Mihi and H. Míguez* \\ Instituto de Ciencia de Materiales de Sevilla, CSIC, Avenida Américo Vespucio s/n, Isla de La Cartuja, 41092 Sevilla, Spain \\ I. Rodríguez, S. Rubio, and F. Meseguer \\ Centro Tecnológico de Ondas, Unidad Asociada CSIC-UPV, Edificio I-2, Universidad Politécnica de Valencia, Camino de Vera s/n, \\ 46022 Valencia, Spain
}

(Received 22 February 2004; revised manuscript received 24 June 2004; published 31 March 2005)

\begin{abstract}
Herein we report an experimental and theoretical optical analysis of the effect of growing a dielectric slab on the surface of photonic colloidal crystals. Optical spectroscopy shows an enhancement of the transmitted intensity for certain frequencies within the photonic pseudogap. Simulations based on a scalar wave approximation fairly reproduce the experimental results and provide a description of the interplay between the features arising from the presence of the surface slab and the finite size of the photonic crystal. The experimental observations are explained by the excitation of photon resonant states at the crystal boundary. Our work demonstrates that the amplitude of light waves penetrating the crystal with frequencies lying within the pseudogap range can be greatly modified by rather simple means.
\end{abstract}

DOI: 10.1103/PhysRevB.71.125131

PACS number(s): 42.70.Qs

The controlled introduction of defects in photonic crystals is a subject of great interest because of the important technological implications it may have in fields such as optoelectronics and telecommunications. ${ }^{1-3}$ Efforts to understand their effect on the optical properties of photonic band gap materials have been done from both the theoretical and the experimental points of view from the early days of the field. ${ }^{4}$ Analogously to what happens when impurities are added to a semiconductor, doping of photonic crystals by adding or removing a dielectric dot, line, or plane gives rise to allowed states for photons with energies lying within the stop bands. Impurity states have been used to guide $\operatorname{light}^{5}$ or for lasing 6 when optically active compounds are introduced in the lattice. Also, photon states in the gap arising as a result of the morphology of the crystal termination and their effect on the wave coupling and diffraction properties have been investigated theoretically and experimentally. ${ }^{7-9}$

A few attempts have been made to dope colloidal lattices. The first one was made in a suspension of self-organized charged spheres by adding different amounts of impurities (spheres of a different kind or size), which randomly distribute through the entire face-centered cubic crystal. ${ }^{10}$ The effect of the allowed modes within the pseudogap on the optical properties was observed and analyzed in terms of the ratio between the size and refractive index of the impurities and those of the scatterers in the host lattice. Later, regarding solid, dried colloidal crystals, the introduction of these dot defects was also performed by adding smaller beads during the crystallization process of polystyrene colloids. ${ }^{11}$ A monotonic increase of the transmittance intensity at pseudogap frequencies was observed as the doping level rose, but no clearly distinguishable states in the gap were observed. Lately, using a combination of convective self-assembly and Langmuir-Blodgett techniques, a silica sphere crystal has been doped with a monolayer of larger spheres of the same composition. ${ }^{12}$ Optical analysis confirmed that this embedded monolayer behaved as a two-dimensional defect. In this case the spectral position of the allowed states relative to the edges of the stop band can be tuned by changing the ratio between the host and dopant sphere diameters. Very recently, a method to create dielectric planar defects of controlled thickness within colloidal crystal films has been developed, and their effect on the optical properties analyzed. ${ }^{13,14}$

While all the aforementioned works report on the effect of defects introduced in the bulk of colloidal crystals, no experimental studies, as far as we know, have been made on the effect of modifying the lattice termination on their optical properties. However, thin excess layers of the guest compound are commonly observed when such lattices are infiltrated. If the closure of the outer pores, which allows the guest material or its precursor to enter the void lattice of the structure, takes place before the infiltration process is finished, an external coating covering the whole structure is formed. In fact, strong modifications of the photonic crystal properties associated with an outer polymer layer in a silicapolymer colloidal crystal composite have recently been reported. ${ }^{15}$ Despite the importance it might have for the monitoring of infiltration processes or the possibility it offers to modify the photonic crystal properties in a controlled manner, an experimental and theoretical study of the effects of such overlayer on the optical properties was still missing.

Here we present an experimental and theoretical analysis of the optical properties of a colloidal photonic crystal having a thin dielectric coating on one of its surfaces. We have performed a systematic study by building up étalons of different thickness and compounds on top of colloidal photonic crystal films, and demonstrated that the optical properties at energies at both the stop and transmission bands are greatly modified by the presence of the surface dielectric slab. The fine features observed in the spectra are well reproduced by simulations based on a scalar wave approximation, which counts for the effect on the reflectance of both the finite crystal size and the presence of a surface layer. The existence of photon resonant states at the modified crystal boundary is demonstrated. 


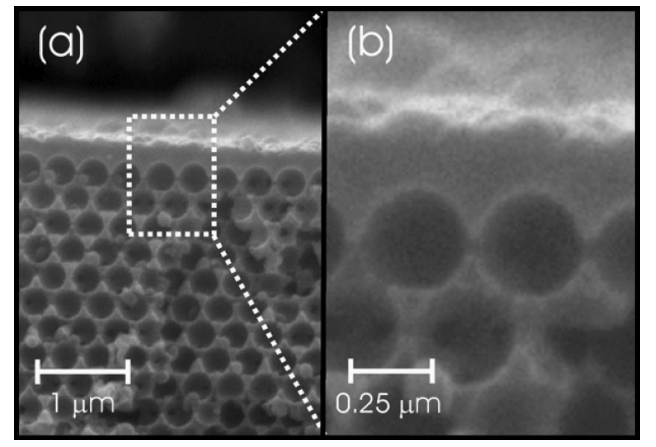

FIG. 1. SEM images of an inverted silica colloidal crystal on which an external homogeneous silica layer has been grown.

Colloidal lattices on which a layer was deposited on top were made using a procedure described in detail elsewhere and which combines convective self-assembly, as the colloidal crystallization technique, with a metal oxide chemical vapor deposition (CVD) method recently developed. ${ }^{16}$ In brief, a colloidal crystal of controlled thickness is built by placing vertically a glass slide in a beaker containing a monodisperse suspension of polystyrene spheres in ethanol. After this, the interstitial voids of the photonic lattice are infiltrated by growing silicon oxide onto the particle surfaces by alternate CVD of water and silicon tetrachloride $\left(\mathrm{SiCl}_{4}\right)$. The infill is gradually increased until the maximum degree of infiltration is achieved, which corresponds to $86 \%$ of the void volume in a layer-by-layer infiltration process of a cubic close-packed lattice. At that point, further infiltration results in an external coating of the crystal whose thickness can be controlled by the number of deposition cycles performed. In order to analyze the effect of the dielectric contrast, the latex template was removed and a lattice of interconnected hollow silica shells coated by an external silica slab was attained. Also with this purpose, high-dielectric-constant compounds, such as titanium oxide and silicon, were infiltrated in silica colloidal crystals. Titanium oxide was grown by CVD, replacing $\mathrm{SiCl}_{4}$ with $\mathrm{TiCl}_{4}$ in the synthetic procedure already described, while silicon was deposited by thermal decomposition of disilane gas. ${ }^{17}$ For both oxides and semiconductors, the use of CVD techniques for infiltration allow control over the filling fraction and the thickness of the external layer. Scanning electron microscope (SEM) images exemplifying the kind of photonic structures that are the subject of our research are shown in Fig. 1.

In order to analyze the effect of the external dielectric slab on the optical properties of colloidal photonic crystals, optical characterization was performed by using microspectroscopy in the visible and near-infrared region. We utilized a microscope attached to a Fourier transform infrared spectrophotometer (model IFS-66, Bruker) to measure the specular reflectance of the different samples. A $\times 4$ objective having a numerical aperture of 0.1 (light cone angle $\pm 5.7^{\circ}$ ) was used to irradiate the sample and collect the reflected light at quasinormal incidence with respect to the (111) planes of the colloidal lattice. A spatial filter was used to selectively detect light from $0.1 \mathrm{~mm}^{2}$ circular regions of the sample.

Reflectance spectra for crystals having dielectric layers on their surface are shown in Figs. 2 and 3. Figure 2(a) shows

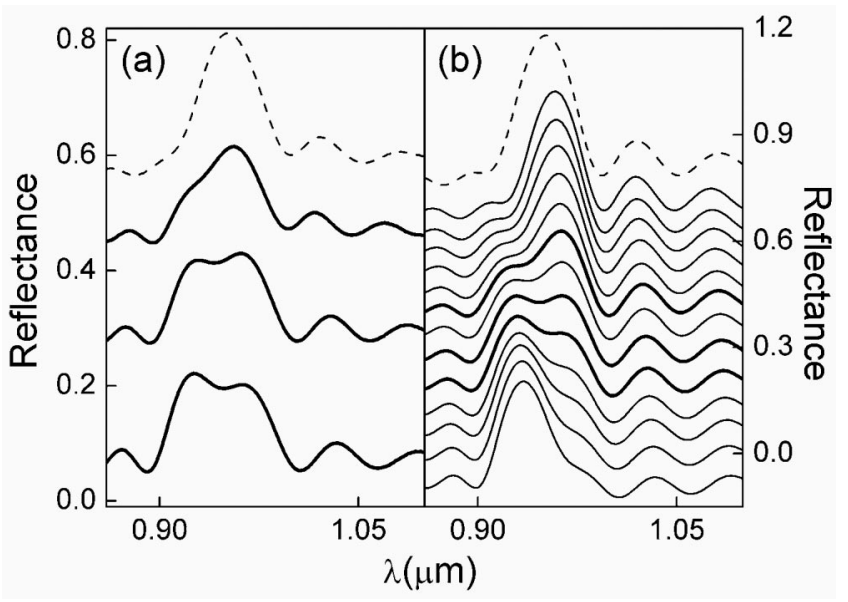

FIG. 2. Specular reflectance for samples having a fixed crystal size and variable surface slab thickness. (a) Specular reflectance spectra of silica-infiltrated polystyrene sphere colloidal crystal films built on top of a glass slide and having a silica slab deposited on top. From top to bottom, slab thickness is $D=0$ (dotted line), 405, 420 , and $430 \mathrm{~nm}$ (solid lines). All crystals are made of the same spheres (diameter $\phi=375 \mathrm{~nm})$ and have the same size $(T=20$ sphere layers). (b) Simulation showing the evolution of the peak shape for a set of fcc lattice made of 20 close-packed layers of $n=1.59$ refractive index spheres (latex) each one with the same degree of infiltration (pore filling of $60 \%$ ) of a material of $n=1.45$ (silica) but having a different dielectric slab of $n=1.45$ (silica) on top. From top to bottom, solid lines correspond to slab thickness ranging from $D=350$ up to $470 \mathrm{~nm}$, with $\Delta D=10 \mathrm{~nm}$. The dotted line represents the curve for a crystal with no surface defect on top. Thick lines are used to highlight those spectra that fit the experimental results. Spectra have been shifted for the sake of clarity.

the results for different latex sphere lattices infiltrated with silica, each one having an excess silica surface layer of slightly different thickness. The spectrum obtained from a silica-filled lattice crystal having no surface layer is also included for comparison in Fig. 2(a) (dotted line). A clear maximum reflects the opening of a pseudogap at the $L$ point of the face-centered cubic sphere lattice under study; the lower-intensity fringes detected on both sides of the main peak are the result of the interference of the beams reflected at the top and bottom surfaces of the photonic crystal film and therefore stem from the finite size of the crystal. The dependence of the optical properties of the pseudogap on crystal thickness and the nature of the substrate has been thoroughly analyzed before and will not be discussed here. ${ }^{18}$ It must be noted, however, that the samples under study are just a small number of periods thick and their dielectric modulation is weak, which causes the reflected intensity at the pseudogap to be lower than 1 . We will focus on the modification of these properties resulting from the presence of the coating. The optical characterization results depicted in Fig. 2(a) show that the reflectance maximum shape is distorted by the presence of the external slab. A dip in the reflectance maximum is typically observed. Simulated spectra were calculated using a scalar wave approximation (SWA), which has been thoroughly analyzed before. ${ }^{19,20}$ This approximation has provided a fair description of the optical 


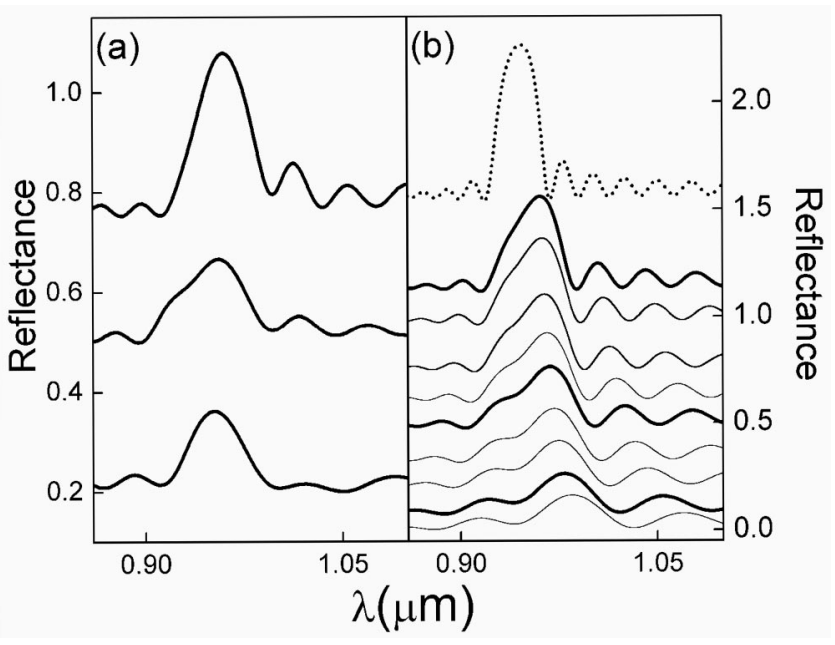

FIG. 3. Specular reflectance for samples having a fixed surface slab thickness and variable crystal size. (a) Specular reflectance spectra of silica-infiltrated polystyrene sphere colloidal crystal films having an external silica slab on top of $D \approx 405 \mathrm{~nm}$ thickness. From top to bottom, the crystal size is $T=30,22$, and $16 \mathrm{ML}$. All crystals are made of the same spheres (diameter $\phi=375 \mathrm{~nm}$ ). (b) Simulation showing the evolution of the peak shape for a set of fcc lattice of $n=1.59$ refractive index spheres (latex) each one with the same degree of infiltration (pore filling fraction of 60\%) of a material of $n=1.45$ (silica) and having the same 405-nm-thick dielectric slab of $n=1.45$ (silica) on top. From bottom to top, solid lines correspond to crystal size ranging from $T=14$ up to $30 \mathrm{ML}$, with $\Delta T=2 \mathrm{ML}$. The dotted line represents the curve for a crystal made of $45 \mathrm{ML}$ and the same $405 \mathrm{~nm}$ slab on top. Thick lines are used to highlight those spectra that fit the experimental results. Spectra have been shifted for the sake of clarity.

properties of photonic crystal superlattices ${ }^{21}$ and doped photonic crystals. ${ }^{13}$ The structure considered in the simulations consist of a planar dielectric slab (refractive index $n=1.45$ ) placed onto the (111) surface of a face-centered cubic sphere $(n=1.59)$ crystal infiltrated with silica $(n=1.45)$ and located on top of a glass substrate $(n=1.5)$. In Fig. 2(b), the calculated spectra obtained from an infiltrated crystal made of 20 sphere layers presenting a slab on top whose thickness varies from $D=350 \mathrm{~nm}$ (top spectra) up to $D=470 \mathrm{~nm}$ (bottom) are drawn. The external slab width difference between consecutive spectra is $\Delta D=10 \mathrm{~nm}$. These simulations reproduce the experimental results and show that the broad dip observed experimentally starts to appear at short wavelengths and moves slowly toward the long-wavelength end of the maximum as the planar defect width increases. This effect repeats quasiperiodically for either larger or smaller defect widths.

The effect of the crystal size on the observed reflectance modulations introduced by the surface étalon was studied by fabricating crystals made of a different number of latex sphere layers, but having a similar degree of silica infiltration (pore filling fraction around 60\%) and an external slab of similar thickness ( $D$ around $405 \mathrm{~nm}$ ) grown on top. Figure 3(a) displays the reflectance from these crystals, whose size increases from $T=16$ sphere monolayers (ML) (bottom curve) to $T=22 \mathrm{ML}$ (middle) and $T=30 \mathrm{ML}$ (top). Figure 3(b) displays different spectra calculated by the SWA, exem-

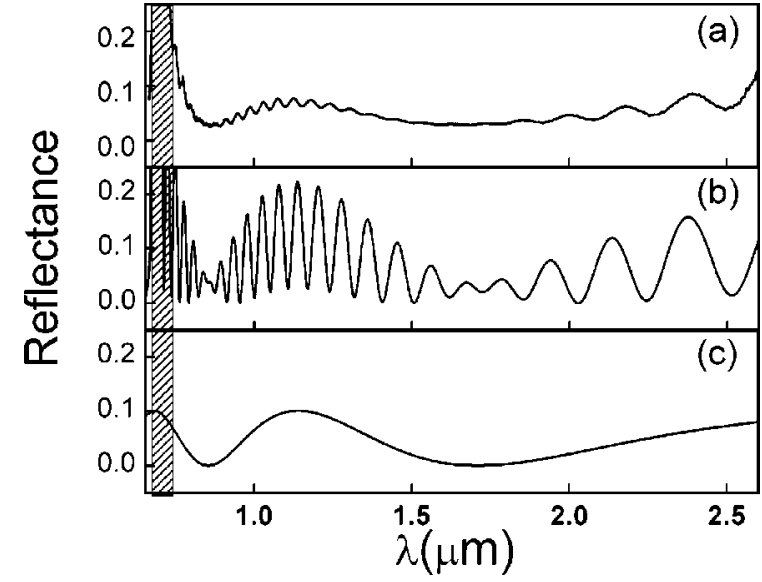

FIG. 4. (a) and (b) show, respectively, the experimental and theoretical specular reflectance curves of an inverted silica colloidal crystal film (hollow cavities of diameter $\phi=375 \mathrm{~nm}$ ) having a silica slab of $D=600 \mathrm{~nm}$ on top. (c) displays the calculated reflectance of a $600 \mathrm{~nm}$ thick silica étalon sandwiched between air and a material of $n=1.05$ (effective refractive index of the photonic crystal in the long-wavelength region).

plifying, from bottom to top, the evolution of the reflectance as we gradually change the crystal size between $T=14$ and 30 ML keeping the surface slab width constant. The dotted line corresponds to a crystal of $T=45 \mathrm{ML}$. As the crystal thickness increases, the depth of the dip is gradually reduced until it completely disappears for very large samples. This is, very likely, the reason why these modifications of the optical features at pseudogap frequencies have not been observed before if an excess layer was accidentally created when infiltrating large colloidal crystals obtained by sedimentation methods, although the large amount of intrinsic defects they present might have obscured its effect in some cases.

A similar experimental and theoretical analysis was performed for the corresponding inverted samples, that is, the colloidal crystals attained after removing the original latex template by chemical etching. This gives rise to a periodic lattice of hollow spherical silica shells. In this case, no dip was observed in the frequency range corresponding to the pseudogap, in good agreement with the simulations. However, the presence of an external slab not only introduces modifications in the pseudogap, but also is able to modulate the reflected light intensity in the passband frequency ranges. This effect could be clearly observed in the inverted silica colloidal crystal films. The experimental and theoretical reflectance are shown in Figs. 4(a) and 4(b), respectively. It can be seen that at wavelengths other than those within the pseudogap spectral range (shaded area in Fig. 4), the reflectance is smoothly modulated. This modulation is caused by the external étalon, whose calculated reflection is drawn in Fig. 4(c). For this calculation, we assume a dielectric $\left(n_{D}=1.45\right)$ thin film $(600 \mathrm{~nm})$ confined between two semiinfinite media, the incident one of $n_{i}=1$ and the substrate in which $n$ equals the volume average refractive index of the colloidal crystal $\left(n_{\mathrm{CC}}=1.05\right)$. By doing this, the finitecrystal-size effects are not considered and only the slab is taken into account. The modulation described here is also 


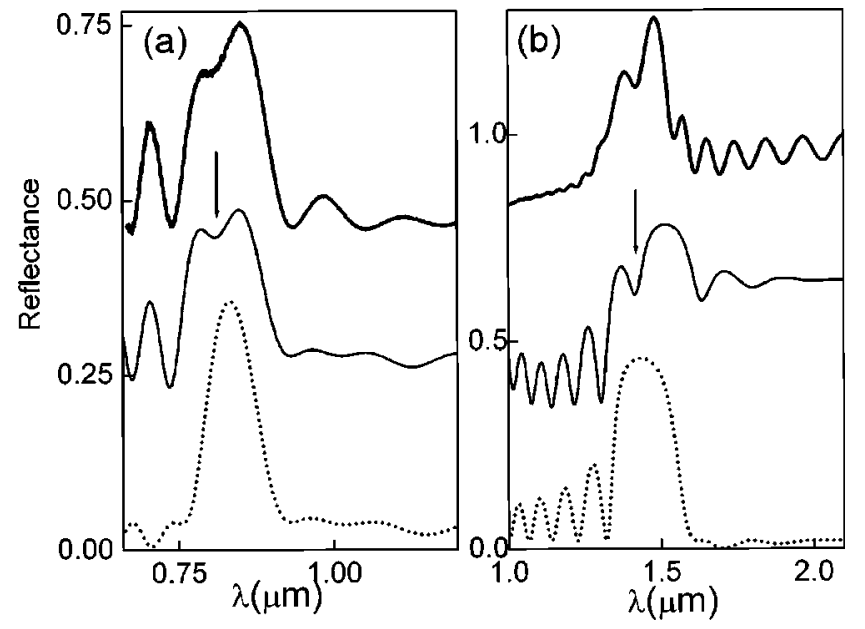

FIG. 5. Experimental (top curve) and calculated (middle curve) specular reflectance spectra for titania- and silicon-infiltrated silica lattices having a surface layer deposited on top. (a) $\mathrm{TiO}_{2}(n=2$, in our case) infiltrated silica $(n=1.43)$ colloidal crystal (sphere diameter $\phi=378 \mathrm{~nm}$ ) with the following relevant parameters: crystal thickness $T=9 \mathrm{ML}, \mathrm{TiO}_{2}$ pore filling fractions $5 \%$, and $\mathrm{TiO}_{2}$ surface layer thickness $D=20 \mathrm{~nm}$. (b) Amorphous Si $(n=3.8$, in our case) infiltrated silica colloidal crystals (sphere diameter $\phi=526 \mathrm{~nm}$ ), $T=11 \mathrm{ML}$, Si filling fraction $30 \%$, and $D=150 \mathrm{~nm}$. In both panels, bottom curves (dotted lines) are the calculated spectra for model structures with the same parameters but with no surface layer on top $(D=0)$. In all cases, spectra have been shifted for the sake of clarity. In both cases, an arrow points the dip in the reflectance maximum.

observed in the lattices prior to inversion, but it is weaker due to the low dielectric contrast between the slab and the averaged crystal $\left(n_{\mathrm{CC}}=1.49 \approx n_{D}=1.45\right)$.

We also studied the effect of having a high-dielectricconstant defect grown on a high-dielectric-contrast lattice. For this purpose, titania $\left(\mathrm{TiO}_{2}\right)$ and amorphous silicon films were deposited on top of lattices of silica spheres partially infiltrated with amorphous silicon or titania, respectively. Figure 5 displays the experimental spectra (thick solid lines) obtained for two different samples of this kind, each one possessing a different crystal and external slab thickness. Simulated spectra are also shown for the corresponding structures with (thin solid lines) and without (dotted lines) an external layer on top. Comparison between these two latter curves highlights the strong effect of the surface layer. In this case, as in the case of the lower-dielectric-contrast composites previously discussed, the reflected intensity is modified at both the stop and the passband regions by the presence of the external layer. However, in this case, the effect is more dramatic as a result of the higher refractive index of the layer. In addition, the reflectance maximum width increases, a feature that is characteristic of photonic crystals in which defects are introduced. ${ }^{10}$ It should be noted that in the case of the silicon-infiltrated crystal, both the intensity and the width of the reflectance maximum observed at pseudogap frequencies are smaller than the values SWA predicts [see thick and thin solid lines in Fig. 5(b)]. Furthermore, the number and intensity of secondary lobes are not so well reproduced by the simulations as for the latex-silica composites. This is

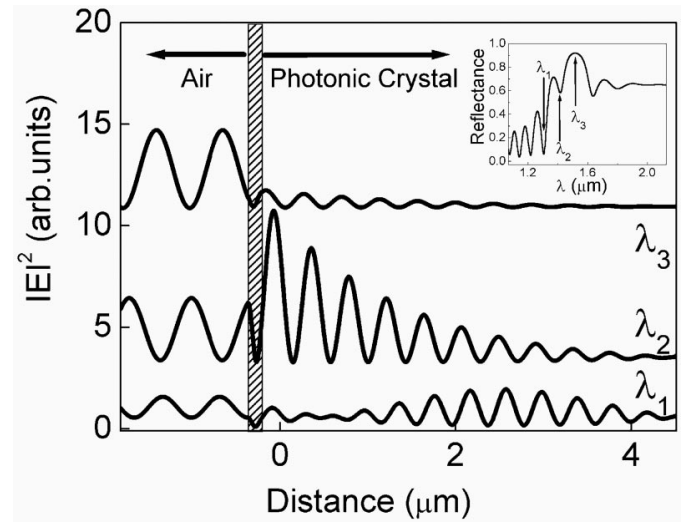

FIG. 6. Spatial variation of the calculated squared magnitude of the electric field for three waves of different wavelength impinging onto a silica sphere $(n=1.43$, sphere diameter $\phi=526 \mathrm{~nm}, T=11$ ML) crystal infiltrated with amorphous silicon $(n=3.8$, pore filling fraction 30\%) and having a $D=150 \mathrm{~nm}$ thick layer of $n=3.8$ on top. Wavelengths are $\lambda_{1}=1.304 \mu \mathrm{m}, \lambda_{2}=1.414 \mu \mathrm{m}$, and $\lambda_{3}=1.518 \mu \mathrm{m}$. All frequencies are indicated with an arrow in the spectrum shown in the inset. The shaded area indicates the location of the surface layer.

probably due to the fact that the effect of intrinsic defects is also amplified as they are infiltrated with a high-dielectricconstant material like silicon. Nevertheless, the effect of the external layer is qualitatively well described by the simulations not only at the stop band frequency range, but also at wavelengths corresponding to the lower-energy passband (dielectric band), in which a higher reflectance than in the high-energy passband (air band) is measured.

In order to visualize the spatial distribution of the wave amplitude in photonic colloidal crystals at the frequencies of interest, we have plotted the squared amplitude of the electric field $|\boldsymbol{E}|^{2}$ calculated using the SWA versus distance along the [111] direction of the colloidal lattice. We have chosen the middle curve shown in Fig. 5(b) as an example, since it displays the deepest dip among all structures studied. For comparison, we have calculated the profile of $|\boldsymbol{E}|^{2}$ for (1) a wavelength in the dielectric passband range; (2) the wavelength at which the dip in the pseudogap reaches its minimum value; (3) a wavelength that lies within the pseudogap and for which the reflectance is maximum $\left(\lambda_{1}, \lambda_{2}\right.$, and $\lambda_{3}$, respectively). Results are shown in Fig. 6 . It can be seen that, while the electric field corresponding to a mode in the pseudogap $\left(\lambda_{3}\right)$ just decays exponentially as we move away from the surface, the mode at which the dip is observed $\left(\lambda_{2}\right)$ presents its maximum intensity at the photonic crystal surface, very close to the external slab-crystal interface. This indicates that the observed dips are the result of the appearance of resonant modes, partially localized at the lattice surface as a consequence of the presence of the external coating. Qualitatively similar results are attained for the latex-silica composites, although the waves are more weakly localized than in the silicon infiltrated samples, which results in the low depth and broad dips observed. This is explicitly shown in Fig. 7, in which $|\boldsymbol{E}|^{2}$ versus distance is plotted for a latex sphere crystal infiltrated and coated with an excess layer of $\mathrm{SiO}_{2}$. The three wavelengths chosen for these calculations 


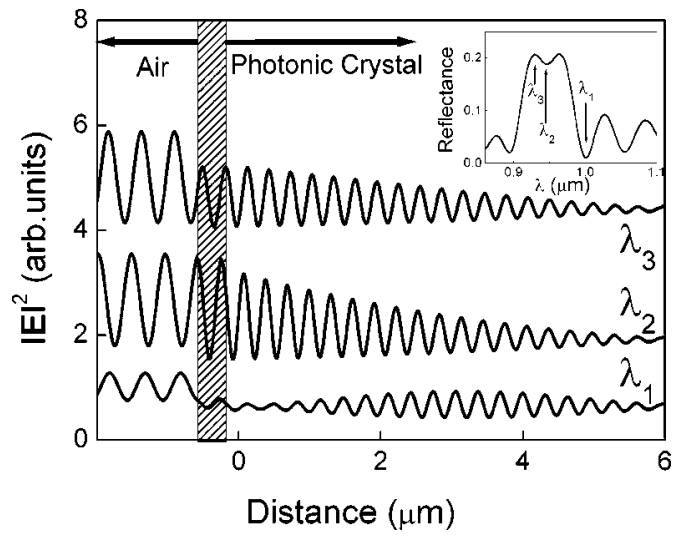

FIG. 7. Spatial variation of the calculated squared magnitude of the electric field for three waves of different wavelength impinging onto a latex sphere $(n=1.59$, sphere diameter $\phi=374 \mathrm{~nm}, T=20$ ML) crystal infiltrated with silicon oxide ( $n=1.45$, pore filling fraction $60 \%$ ) and having a $D=430 \mathrm{~nm}$ thick layer of $n=1.45$ on top. Wavelengths are $\lambda_{1}=0.876 \mu \mathrm{m}, \lambda_{2}=0.945 \mu \mathrm{m}$, and $\lambda_{3}=0.929 \mu \mathrm{m}$. All frequencies are indicated with an arrow in the spectrum shown in the inset. The shaded area indicates the location of the surface layer.

are pointed out in the reflectance spectrum corresponding to the sample under analysis shown in the inset of Fig. 7. Only a comparison between $|\boldsymbol{E}|^{2}$ versus distance for $\lambda_{2}$ and $\lambda_{3}$ allows us to claim the existence of a weak resonance for $\lambda_{2}$, which in this case takes place within the slab.

From what we have just exposed, it can be inferred that we can regard the excess layer as a type of surface dopant, since it introduces allowed states in the forbidden photonic bands. The magnitude of the electric fields under study is maximum at a shallow depth very close to the surface of the slab-crystal system. These surface modes are responsible for the appearance of the dip in the reflectance spectrum observed when the number of periods in the structure is finite, since the tail of the corresponding evanescent wave propagating in the perpendicular direction away from the surface may couple with a mode in the substrate. However, it should be remarked that the wave vector of such modes has no component parallel to the surface $\left(k_{\|}=0\right)$ and therefore does not oscillate along that direction. Also, as shown in Figs. 6 and 7, these states are extended in the incoming medium (air) and decaying inside the crystal. Therefore, they are not strictly localized at the surface, regarding as such those that decay on both sides of the interface and oscillate in the direction parallel to it. ${ }^{22}$ That type of mode has been observed in truncated one-dimensional optical multilayers. ${ }^{23}$ It is interesting to notice that transmission enhancement by surface resonant states that can be coupled by normally incident waves $\left(k_{\|}=0\right)$ has been previously reported for acoustic superlattices in the frequency passband region. ${ }^{24}$ Elastic guided modes supported by a surface layer on a two-dimensional acoustic crystal have also been studied. ${ }^{25}$

Since the effect of the surface layer is stronger for the case of the silicon-infiltrated silica sphere crystals, we have chosen this composite as a model to theoretically study the variation of the wavelength at which the local reflectance minimum is observed within the pseudogap range, $\lambda_{S}$, with

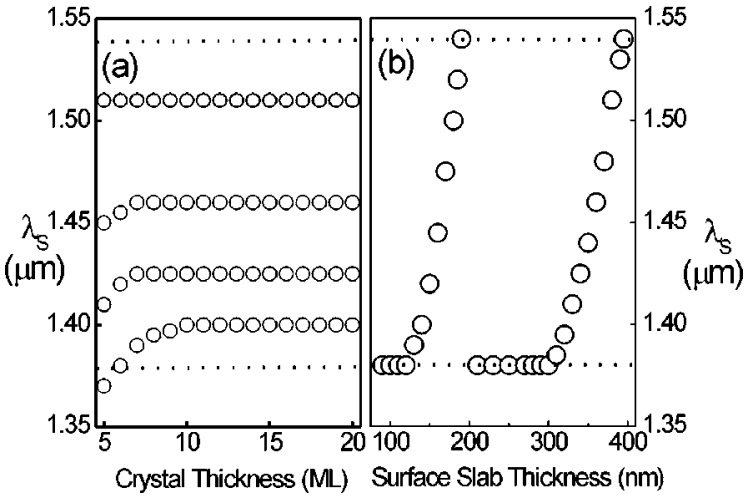

FIG. 8. (a) Variation of the calculated spectral position of the surface mode $\lambda_{S}$ with the crystal thickness (in sphere monolayers) for structures having slabs of different widths $D$ on top. From bottom to top, $D=320,340,360$, and $380 \mathrm{~nm}$. (b) $\lambda_{S}$ versus surface slab thickness for a fixed crystal size (20 monolayers). All calculations have been performed for a silica sphere $(n=1.43$, diameter $\phi=526 \mathrm{~nm})$ crystal infiltrated with amorphous silicon $(n=3.8$, pore filling fraction $30 \%$ ), and having a layer of $n=3.8$ and width $D$ on top.

the different parameters of the layer-crystal structure. Figure 8 shows the evolution of $\lambda_{S}$ as both the surface slab and the photonic crystal widths are varied. In Fig. 8(a), each scatter graph for $\lambda_{S}$ versus photonic crystal width corresponds to a different silicon slab thickness, which increases from the bottom curve to the top one from 320 to $380 \mathrm{~nm}$ with a step of $20 \mathrm{~nm}$. The dotted lines represent the edges of the photonic pseudogap calculated for a 100-sphere-monolayer-thick silicon-infiltrated silica crystal. Several interesting features are observed. For a fixed slab thickness, $\lambda_{S}$ increases with the crystal size in the range of very thin crystals, reaching a constant value for crystals larger than a few sphere monolayers. This dependence is strong for surface modes in the range close to the air band and is almost absent for those centered in the pseudogap region or close to the dielectric band. This latter result can be understood if we consider an effective confining distance determined by the slab width plus a certain depth of the crystal beyond which we can regard the wave as totally attenuated. Our calculations show that for thin crystals, among waves whose frequencies lie within the pseudogap, those close to the high-energy edge (air band) decay more slowly with distance than those close to the lowenergy end (dielectric band). Thus, for a fixed crystal size, the effective confining distances are larger for the former than for the latter, as is the crystal size needed to reach the same level of attenuation. In all cases, no further significant effect of the crystal size on the partially localized mode spectral position $\lambda_{S}$ or magnitude $|\boldsymbol{E}|^{2}$ is observed for lattices larger than ten sphere monolayers. To avoid any effect due to the crystal size, a crystal of 20 sphere monolayers was used to calculate the $\lambda_{S}$ versus surface slab thickness relation plotted in Fig. 8(b). For a fixed crystal size, $\lambda_{S}$ sweeps the pseudogap region from the air band edge to the dielectric band edge as more dielectric is added to the surface, as expected for any resonant mode as the boundaries of the confining cage become further apart. For certain slab thickness ranges no modes supported by the slab-crystal system lie 

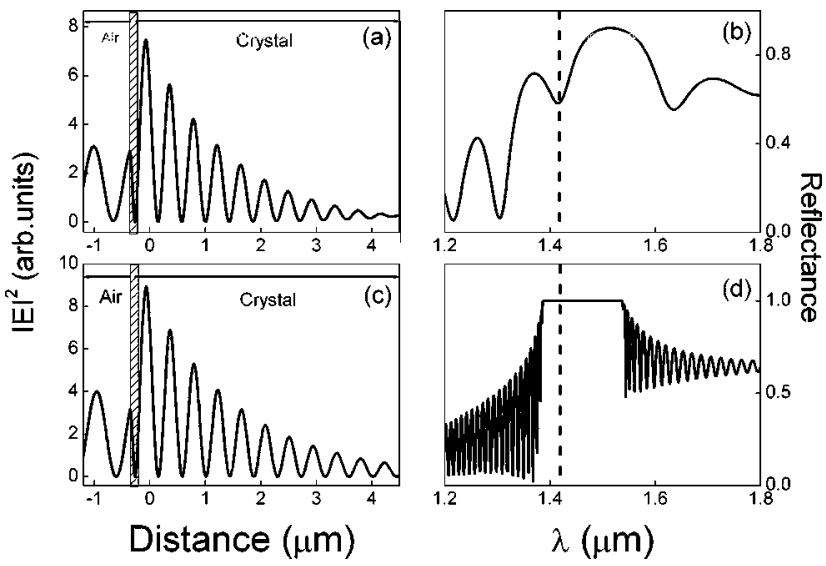

FIG. 9. (a) Calculated spatial distribution of the squared magnitude of the electric field for a silica sphere $(n=1.43$, sphere diameter $\phi=526 \mathrm{~nm}, T=11 \mathrm{ML}$ ) crystal infiltrated with amorphous silicon ( $n=3.8$, pore filling fraction $30 \%)$ and having a $D=150 \mathrm{~nm}$ thick layer of $n=3.8$ on top. The frequency at which the electric field is calculated is that at which a dip in the reflectance spectrum is observed, as indicated with a dotted line in (b). The same sort of calculation was performed at that same wavelength for a 100-layerthick crystal of similar composition and having the same slab on the surface. Results are displayed in (c) and (d).

within the pseudogap spectral window. It should be noticed that the existence of a surface resonance caused by the presence of a dielectric slab does not imply necessarily the detection of a dip in the reflectance spectrum. For instance, for the system modeled in Fig. 8, once the crystal size is increased above a few sphere monolayers, the spatial distribution of $|\boldsymbol{E}|^{2}$ for the surface mode remains almost unaltered. At the same time, as the crystal becomes thicker, the coupling of the surface mode with the substrate modes is reduced and so is the depth of the reflectance maximum dips. Eventually, no modification is observed in the reflectance spectrum although there exists a resonant mode at the surface. This is explicitly shown in Fig. 9, in which we have plotted the calculated spatial distribution of $|\boldsymbol{E}|^{2}$ and the reflectance spectra for two photonic crystals made of a different number of sphere layers but having a dielectric layer of the same thickness on their surfaces. The wavelength chosen for this comparison is that at which a minimum of the reflectance dip is observed for the 11-layer-thick crystal [dotted vertical line in Figs. 9(b) and 9(d)]. We have also calculated the spatial distribution of $|\boldsymbol{E}|^{2}$ for different wavelengths in order to evaluate the spectral width of the surface resonance. The spectral variation of the maximum value of $|\boldsymbol{E}|^{2}$ versus distance is plotted in Fig. 10 for both an 11- (open circles) and a 100-layer (black circles) thick crystal. It can be seen that, even when the reflectance spectrum does not show any evidence of the presence of a surface slab for the thicker crystal, the spectral shape of the surface resonance for that crystal resembles that of the thinner one, although it is slightly sharper.

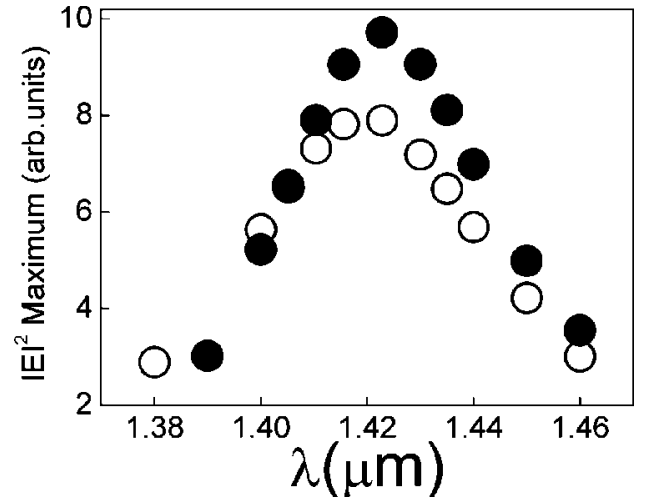

FIG. 10. Spectral variation of the maximum of the $|\boldsymbol{E}|^{2}$ versus distance curve for two silica sphere $(n=1.43$, sphere diameter $\phi=526 \mathrm{~nm})$ crystals infiltrated with amorphous silicon $(n=3.8$, pore filling fraction $30 \%$ ) and having a $D=150 \mathrm{~nm}$ thick layer of $n=3.8$ on top. Open circles correspond to the results obtained for a $T=11 \mathrm{ML}$ thick crystal and black circles to those obtained for a $T=100 \mathrm{ML}$ thick crystal.

Regarding the technological implications of our study, it should be noted that the surface modes described in this work are very sensitive to small variations of the slab thickness. A small difference $(\sim 10 \mathrm{~nm})$ in the thickness of the slab may substantially modify the spatial distribution of the electric field at the surface and, if the crystal is thin enough, its spectrum, as was experimentally and theoretically shown in Fig. 2. This high sensitivity can be put into practice in devices based on the modification of the outer layer, as has been proposed before for truncated $1 \mathrm{D}$ periodic multilayers. ${ }^{9}$

In conclusion, we have analyzed both experimentally and theoretically the effect on the optical properties of having a surface dielectric slab deposited on top of colloidal photonic crystals. We have demonstrated that this layer gives rise to a modulation of the scattered light intensity at both the stop and the passband energy regions, providing a systematic analysis of this effect as a function of the crystal size, surface layer thickness, and dielectric constants of the composite materials. Furthermore, we have proved that the external layer causes the appearance of resonant photon states at the modified crystal surface at frequencies lying within the stop band of the structure. The high sensitivity of these modes to the thickness and refractive index of the surface defect could be useful for monitoring the deposition process of thin films or building up sensing surface devices based on their modification by an external agent.

This work has been partially funded by Fundación Ramón Areces, under grant "Colloidal Photovoltaic Materials" and the Spanish CICyT, under Grant No. MAT2003-04993-C0401. I.R. thanks the Ministry of Science and Technology for a "Ramón y Cajal" fellowship. H.M. and A.M. thank A. Hakansson and J. Sánchez-Dehesa for fruitful conversations. 
*Corresponding author. Electronic address: hmiguez@icmse.csic.es

${ }^{1}$ S. John, Phys. Rev. Lett. 58, 2486 (1987).

${ }^{2}$ E. Yablonovitch, Phys. Rev. Lett. 58, 2059 (1987).

${ }^{3}$ J. D. Joannopoulos, P. R. Villeneuve, and S. Fan, Nature (London) 386, 143 (1997).

${ }^{4}$ E. Yablonovitch, T. J. Gmitter, R. D. Meade, A. M. Rappe, K. D. Brommer, and J. D. Joannopoulos, Phys. Rev. Lett. 67, 3380 (1991).

${ }^{5}$ J. C. Knight, J. Broeng, T. A. Birks, and P. St. J. Russell, Science 282, 1476 (1998).

${ }^{6}$ O. J. Painter, R. K. Lee, A. Scherer, A. Yariv, J. D. O’Brien, P. D. Dapkus, and I. Kim, Science 284, 1819 (1999).

${ }^{7}$ G. von Freymann, W. Koch, D. C. Meisel, M. Wegener, M. Diem, A. García-Martín, S. Pereira, K. Busch, J. Schilling, R. B. Wehrspohn, and U. Gösele, Appl. Phys. Lett. 83, 614 (2003).

${ }^{8}$ L. L. Lin and Z. Y. Li, Phys. Rev. B 69, 193103 (2004).

${ }^{9}$ F. Villa, L. E. Regalado, F. Ramos-Mendieta, J. Gaspar-Armenta, and T. López-Ríos, Opt. Lett. 27, 646 (2002).

${ }^{10}$ R. D. Pradhan, I. I. Tarhan, and G. H. Watson, Phys. Rev. B 54, 13721 (1996)

${ }^{11}$ B. Gates and Y. Xia, Appl. Phys. Lett. 78, 3178 (2001).

${ }^{12}$ Y. Zhao, K. Wostyn, G. de Schaetzen, K. Clays, L. Hellemans, A. Persons, M. Szekeres, and R. A. Schoonheydt, Appl. Phys. Lett. 82, 3764 (2003).

${ }^{13}$ N. Tetreault, A. Mihi, H. Míguez, I. Rodríguez, V. Kitaev, F. Meseguer, and G. A. Ozin, Adv. Mater. (Weinheim, Ger.) 16, 346 (2004).
${ }^{14}$ E. Palacios-Lidón, J. F. Galisteo-López, B. Hernández-Juárez, and C. López, Adv. Mater. (Weinheim, Ger.) 16, 341 (2004).

${ }^{15}$ A. Arsenault, J. Halfyard, Z. Wang, V. Kitaev, G. A. Ozin, I. Manners, A. Mihi, and H. Míguez, Langmuir 21, 499 (2005).

${ }^{16}$ H. Míguez, N. Tetreault, B. Hatton, S. M. Yang, D. Perovic, and G. A. Ozin, Chem. Commun. (Cambridge) 222736 (2002).

${ }^{17}$ A. Blanco, E. Chomski, S. Grabtchak, M. Ibisate, S. John, S. W. Leonard, C. López, F. Meseguer, H. Míguez, J. P. Mondia, G. A. Ozin, O. Toader, and H. M. Van Driel, Nature (London) 405, 437 (2000).

${ }^{18}$ J. F. Bertone, P. Jiang, K. S. Hwang, D. M. Mittleman, and V. L. Colvin, Phys. Rev. Lett. 83, 300 (1999).

${ }^{19}$ K. W. K. Shung and Y. C. Tsai, Phys. Rev. B 48, 11265 (1993).

${ }^{20}$ D. M. Mittleman, J. F. Bertone, P. Jiang, K. S. Hwang, and V. L. Colvin, J. Chem. Phys. 111, 345 (1999).

${ }^{21}$ P. Jiang, G. N. Ostojic, R. Narat, D. M. Mittleman, and V. L. Colvin, Adv. Mater. (Weinheim, Ger.) 13, 389 (2001).

${ }^{22}$ For a detailed description of the different types of surface states, see J. D. Joannopoulos, R. D. Meade, and J. N. Winn, Photonic Crystals: Molding the Flow of Light (Princeton University Press, Princeton, NJ, 1995).

${ }^{23}$ P. Yeh, A. Yariv, and A. Y. Cho, Appl. Phys. Lett. 32, 104 (1978).

${ }^{24}$ B. Manzanares-Martínez and F. Ramos-Mendieta, Phys. Rev. B 66, 092302 (2002).

${ }^{25}$ B. Manzanares-Martínez and F. Ramos-Mendieta, Phys. Rev. B 68, 134303 (2003). 